\title{
Prioritization and comprehensive analysis of genes associated with melanoma
}

\author{
CHENG FENG, MING BAI, HAILIN ZHANG, ANG ZENG and WENCHAO ZHANG
}

Department of Plastic Surgery, Peking Union Medical College Hospital, Beijing 100730, P.R. China

Received August 13, 2018; Accepted February 28, 2019

DOI: $10.3892 / \mathrm{ol} .2019 .10284$

\begin{abstract}
Melanoma is a malignant tumor derived from melanocytes, which occurs mostly in the skin. A major challenge in cancer research is the biological interpretation of the complexity of cancer somatic mutation profiles. The aim of the present study was to obtain a comprehensive understanding of the formation and development of melanoma and to identify its associated genes. In the present study, a pipeline was proposed for investigating key genes associated with melanoma based on the Online Mendelian Inheritance in Man and Search Tool for the Retrieval of Interacting Genes/Proteins databases through a random walk model. Additionally, functional enrichment analysis was performed for key genes associated with melanoma. This identified a total of 17 biological processes and 30 pathways which may be associated with melanoma. In addition, melanoma-specific network analysis followed by Kaplan-Meier analysis along with log-rank tests identified tyrosinase, hedgehog acyltransferase, BRCA1-associated protein 1 and melanocyte inducing transcription factor as potential therapeutic targets for melanoma. In conclusion, the present study increased the knowledge of melanoma progression and may be helpful for improving its prognosis.
\end{abstract}

\section{Introduction}

Melanoma is the most lethal skin malignancy, and the estimated number of new cases with melanoma of the skin based on conventional cancer incidence rates is 96,480 and the estimated number of cases of mortality is 7,230 for 2019 (1-3). Cutaneous melanoma is the fifth most common type of cancer, with its incidence rate increasing continuously during the past decades $(4,5)$. Despite high diagnostic

Correspondence to: Mr. Ming Bai, Department of Plastic Surgery, Peking Union Medical College Hospital, 1 Shuaifuyuan Road, Dongcheng, Beijing 100730, P.R. China

E-mail: mingbai18201@sohu.com

Key words: melanoma, random walk model, Online Mendelian Inheritance in Man, Search Tool for the Retrieval of Interacting Genes/Proteins, prognosis accuracy and appropriate treatment due to medical advances, its pathogenesis and natural course remain unclear.

Melanoma is a malignant type of tumor, which may be composed of epidermal melanocytes, nevocytes or dermal melanocytes. Epidemiology studies revealed that melanoma is likely to be caused by hereditary factors and environmental risk factors, including sex, age, ethnicity and geographic location $(6,7)$. This is in line with the most vital and potentially reversible risk factor of malignant melanoma arising from an interaction between environmental exposure and genetic susceptibility. Gandini et al (8) revealed the association between melanoma and ultraviolet (UV)-irradiation, and concluded that intermittent sunlight may be a vital determinant of risk. Additionally, other types of UV ray exposure, including sunburn and artificial UV-irradiation, may cause melanoma development $(9,10)$. Out of all melanoma cases, $\sim 25 \%$ occur together with pre-existing moles, suggesting that melanocytes are benign accumulations of themselves and nevus cells (11). A family history may be a strong risk factor for the disease, and provides a direction for elucidating the genetic basis of melanoma $(12,13)$. In addition, melanoma has a high degree of malignancy, and is prone to early lymphoid and blood tract metastasis, which are associated with poor prognosis; therefore, it is important to identify susceptibility genes. Genomic profiling and sequencing will form the basis for molecular taxonomy for more accurate subgrouping of patients with melanoma in the future.

Identification of associated genes is a central challenge of modern genetics aiming to modulate signal pathways and biological processes for a number of well-known diseases, including various types of cancer. Melanoma is highly complicated; therefore, research strategies are limited. Computational methods provide important complementary tools for this problem. Over the past decades, as biotechnology has advanced, a large amount of gene expression data has been collected and submitted to several large-scale databases. These have been extensively used to explore the molecular mechanism of tumorigenesis with bioinformatics analysis methods $(14,15)$. However, a previous study (16) based on sequence- and network-based methods has been conducted to identify melanoma-associated genes.

The purpose of the present study was to establish a comprehensive method to identify melanoma-associated biological mechanisms and to predict key genes associated with melanoma using the random walk method. Gene Ontology (GO) 
terms and Kyoto Encyclopedia of Genes and Genomes (KEGG) pathway enrichment were analyzed to illuminate the biological pathways and processes which may be altered in patients with melanoma. Additionally, network and Kaplan-Meier analyses identified several potential therapeutic targets for melanoma. The results of the present study may provide novel strategies for diagnosis and treatment of melanoma and uncover the probable mechanism of tumorigenesis.

\section{Materials and methods}

Data collection/data set. The present study searched for genes associated with melanoma using the Online Mendelian Inheritance in Man (OMIM) database (https://www.omim. org/), which is a comprehensive, authoritative and timely research resource, and includes descriptions of human genes, phenotypes and their associations (17). A total of 74 melanoma-associated genes were identified. These were used as seed genes for prioritization of melanoma candidate genes in the present study.

The Search Tool for the Retrieval of Interacting Genes/Proteins (STRING) database (http://string-db.org) provides a comprehensive catalogue of protein-protein interactions along with their confidence score according to means obtaining them. In the present study, the full list of gene pairs as background was obtained and the melanoma-associated network was extracted by retaining only the direct neighborhoods of the 74 seed genes, which resulted in a network of 11,181 genes and 39,043 interaction pairs.

Prioritization of melanoma candidate genes. Random walk on heterogeneous networks is an emerging approach for effective disease gene prioritization. Melanoma-associated genes obtained from OMIM and candidate genes in the melanoma-associated network were used as inputs. The random walk algorithm produced a ranked list of the genes, according to their strength of association with melanoma. The risk score of each candidate gene was calculated as follows:

$$
p^{t+1}=(1-r) Q p^{t}+r p^{0}
$$

Where $p^{t}$ represents the melanoma risk vector at walk step $t$, the parameter $r \epsilon(0,1)$ is the restart probability, and $Q$ is the risk probability transfer matrix.

According to the Perron-Frobenius theorem (18), the eigenvalues of stochastic matrix $Q$ are in the range of $[-1,1]$ and $r=0.1$ was finally obtained as the optimal parameter. Generally, the transition probability from gene $q i$ to phenotype $q j$ is defined as:

$$
q_{i j}=\frac{w_{i j}}{\sum_{k \in \text { neighbor }(i)} w_{k j}}
$$

These probabilities can reach a steady state following a sufficiently large number of iterations. The iterations were conducted until the difference between $p_{s}$ and $p_{s+1}$ (measured by the $\mathrm{L}_{1}$ norm) fell below $10^{-6}$.

Functional enrichment analysis. The GO database (http://geneontology.org), which unifies genetic and gene product characteristics of all species, is an effective and efficient tool to screen genes. In the present study, GO functional enrichment analysis of melanoma candidate genes was performed to interpret their biological significance using the WEB-based Gene SeT AnaLysis Toolkit (http://www.webgestalt.org) (19), with a cut-off criterion of $\mathrm{P}<0.05$.

The full list of KEGG pathways was downloaded from the KEGG database (https://www.genome.jp/kegg/), and pathway enrichment analysis for melanoma candidate genes was conducted using the following formula:

$$
p=1-\sum_{j=0}^{m-1} \frac{\left(\begin{array}{c}
M \\
j
\end{array}\right)\left(\begin{array}{c}
N-M \\
n-j
\end{array}\right)}{\left(\begin{array}{l}
N \\
n
\end{array}\right)}
$$

Where $\mathrm{N}$ is the total number of genes in the KEGG pathway database, $n$ is the number of melanoma candidate genes annotated by the KEGG pathway database, $m$ is the number of melanoma candidate genes annotated by a specific KEGG pathway and $\mathrm{M}$ is the total number of genes involved in a specific pathway. KEGG pathways were considered as statistically significant if $\mathrm{P}<0.05$ following correction using the Benjamini-Hochberg method (20).

Pathway crosstalk analysis. Associations among significantly enriched pathways were explored through crosstalk analysis using the overlap coefficient (OC) and the Jaccard coefficient (JC), which were defined as follows:

$$
\begin{gathered}
\mathrm{OC}=\frac{|A \cap B|}{\min (|A|,|B|)} \\
\mathrm{JC}=\left|\frac{A \cap B}{A \cup B}\right|
\end{gathered}
$$

Where $\mathrm{A}$ and $\mathrm{B}$ represent the melanoma candidate gene numbers contained in pathway A and pathway B, respectively. Pathway A and pathway B were considered to be connected if $\mathrm{OC}>0.5$ and JC $>0.25$. Furthermore, Cytoscape 3.0 software (http://cytoscape.org/) was used to visualize connections among KEGG pathways.

Melanoma-specific network analysis. The melanoma candidate genes were uploaded to the STRING database to obtain a melanoma-specific network by using the threshold of confidence score $>0.4$. Additionally, gene degree and betweenness were applied for evaluating gene importance in the network. Khuri and Wuchty (21) hypothesized that MDSets could provide a novel method of evaluating the core proteins of an organism. The present study considered genes contained in the MDSet with a high degree and betweenness as important biomarkers for melanoma progression.

Survival analysis. To evaluate the association among melanoma-associated biomarkers and survival rates, the Gene Expression Profiling Interactive Analysis (GEPIA; http://gepia.cancer-pku.cn) database was used to perform survival analysis. Melanoma samples were classified into high or low expression groups according to the median expression value of specific respective genes. The Kaplan-Meier method and a two-side log-rank test were used for overall survival comparison between two groups. $\mathrm{P}<0.05$ was considered to indicate a statistically significant difference. 


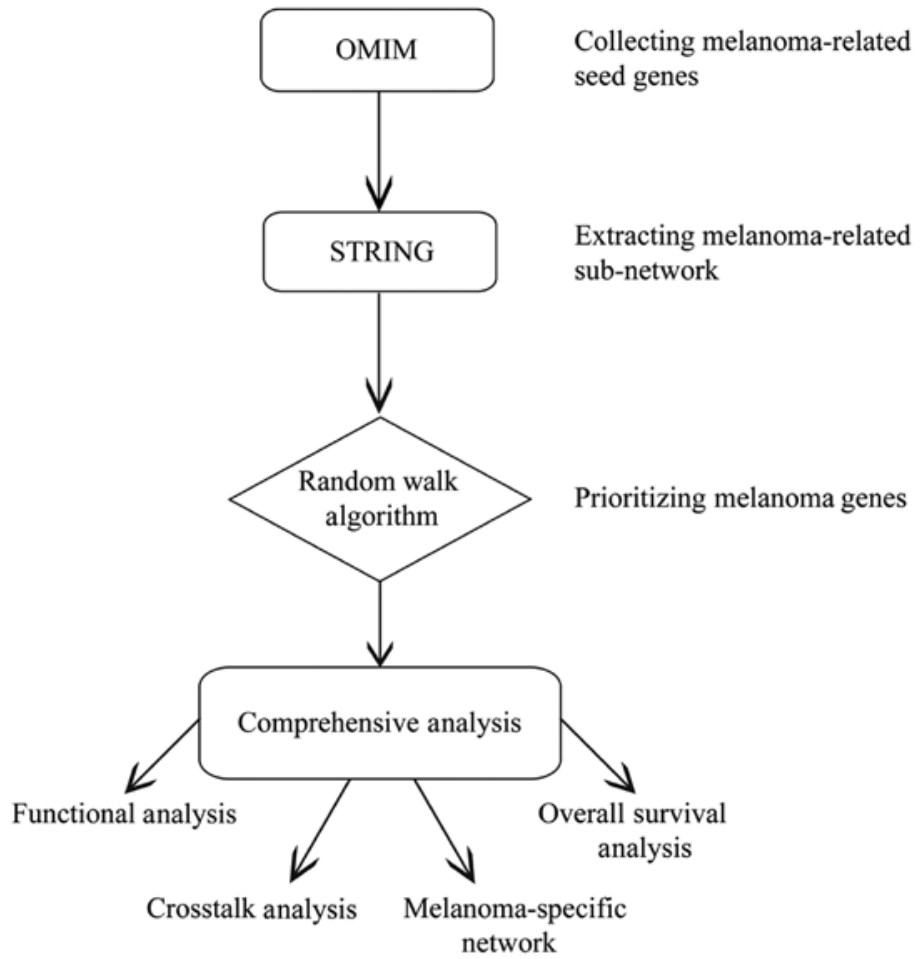

Figure 1. Schematic explaining the workflow of the present study. OMIM, Online Mendelian Inheritance in Man; STRING, Search Tool for the Retrieval of Interacting Genes/Proteins.

Statistical analysis. All statistical analyses were conducted in $\mathrm{R}$ version 3.4.1 (https://www.r-project.org/). For functional analysis, the enrichment method was used. For survival analysis, the Kaplan-Meier method was adopted to plot survival curves, followed by a log-rank test to compare the differences between the survival curves. Data was presented as the mean \pm standard deviation with at least three repeats in every group. $\mathrm{P}<0.05$ was considered to indicate a statistically significant difference.

\section{Results}

Melanoma candidate gene prioritization. Fig. 1 shows the workflow of the present study. A total of 74 genes were reported to be associated with melanoma in the OMIM database, and used as seed genes for following analyses. Additionally, 39,043 interaction pairs among 11,181 genes were obtained from the STRING database by extracting the direct interactions of the 74 seed genes, which was considered as the melanoma-associated network. By applying the random walk algorithm to the 74 seed genes and 11,181 candidate genes, a score was assigned to every candidate gene and the top $1 \%$ of genes $(n=111)$ with the highest scores were considered as melanoma-associated genes.

Significantly enriched functions. The 111 melanoma-associated genes were identified to be significantly enriched in 13 GO biological process (BP) terms, two cellular component (CC) terms and two molecular function terms (Fig. 2A). The BP terms mainly involved in the process of pigmentation, catabolism, telomeres and thymus development. The CC terms melanosome and pigment granule were also considerably enriched. These are involved in pigmentation.
Enrichment Map (http://www.baderlab.org/Software/ EnrichmentMap) was used to explore the associations among significantly enriched GO terms, which resulted in two clusters (Fig. 2B). One cluster included biological processes associated with telomeres and pigmentation, including 'telomere maintenance' and 'pigment cell differentiation', and the other cluster included biological processes associated with catabolism.

There were 30 pathways that were significantly enriched in association with the 111 melanoma-associated genes (Table I), and 12 of them were involved in cancer-associated processes. In addition, endocrine system pathways (melanogenesis and gonadotropin-releasing hormone signaling pathway) and pathways in the translation process [epidermal growth factor receptor signaling pathway and mechanistic target of rapamycin (mTOR) signaling pathway] were also included in the results. The immune system ( $\mathrm{T}$ cell receptor signaling pathway, NOD-like receptor signaling pathway and natural killer cell mediated cytotoxicity) was identified to be overrepresented in melanoma-associated genes. Pathway crosstalk analysis obtained two pathway clusters that were associated with cancer development and the neuroendocrine system, respectively, which were connected by pathways in cancer (Fig. 2C).

Melanoma-specific network. Fig. 3A illustrates the melanoma-specific network that contains 56 melanoma-associated genes and 98 gene pairs. The network MDSet, obtained by using the binary integer linear method, contained 16 genes. Three genes, including tyrosinase (TYR), mitogen-activated protein kinase (MAPK) 3 and cancer/testis antigen 2 (CTAG2), intersected with the top 16 genes with highest betweenness or degree and were considered as important melanoma progression biomarkers. Fig. 3B illustrates associations of genes in 
A



B

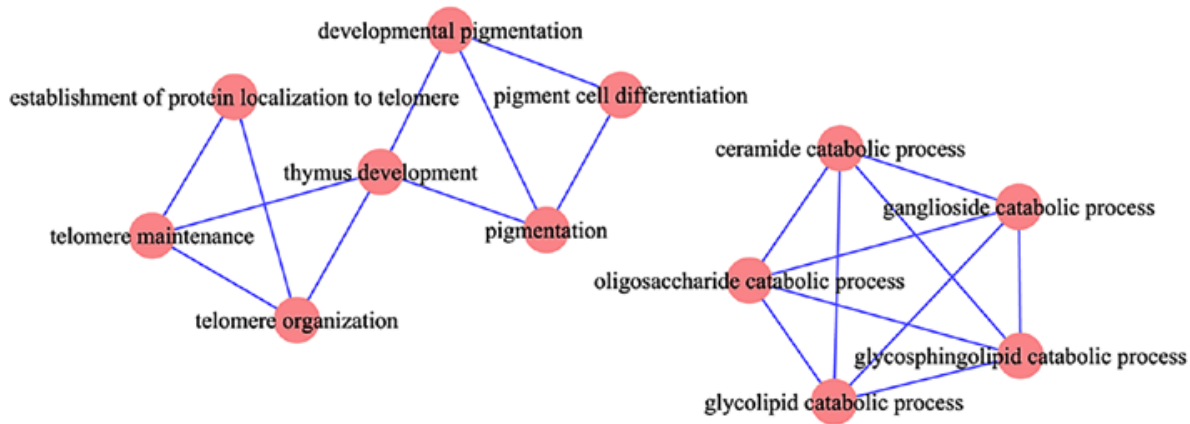

C

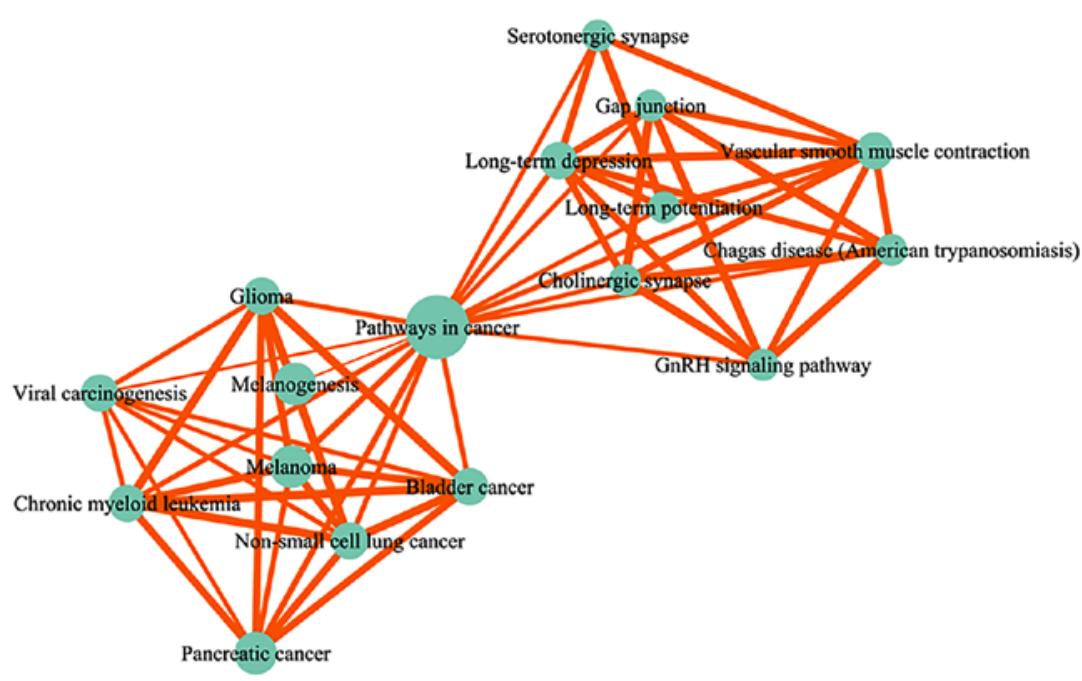

Figure 2. Significantly enriched GO terms and KEGG pathways of 111 melanoma-associated genes. (A) Dot plot illustrating the full list of significantly enriched GO terms and their corresponding logarithm transformed P-values and gene numbers. (B) GO term clustering analysis according to overlapping genes among them. (C) KEGG pathway crosstalk analysis. Nodes represent pathways, and two nodes are connected if there are any overlapping genes between them. Larger node size indicates more genes contained in a pathway, and thicker edges indicate more overlapping genes between two pathways. BP, biological process; CC, cellular component; GnRH, gonadotropin-releasing hormone; GO, Gene Ontology; KEGG, Kyoto Encyclopedia of Genes and Genomes; MF, molecular function. 
Table I. Significantly enriched Kyoto Encyclopedia of Genes and Genomes pathways of the 111 melanoma-associated genes.

\begin{tabular}{|c|c|c|c|}
\hline Pathway & P-value & PBH-value & Melanoma-associated genes included in the pathway \\
\hline Melanoma & $9.45 \times 10^{-7}$ & $1.30 \times 10^{-4}$ & MITF; CDK4; BRAF; MAPK3; MAPK1; CDKN2A \\
\hline Bladder cancer & $1.32 \times 10^{-6}$ & $1.30 \times 10^{-4}$ & CDK4; BRAF; MAPK3; MAPK1; CDKN2A \\
\hline Pancreatic cancer & $6.10 \times 10^{-7}$ & $1.30 \times 10^{-4}$ & CDK4; BRCA2; BRAF; MAPK3; MAPK1; CDKN2A \\
\hline Non-small cell lung cancer & $6.37 \times 10^{-6}$ & $4.21 \times 10^{-4}$ & CDK4; BRAF; MAPK3; MAPK1; CDKN2A \\
\hline Melanogenesis & $7.12 \times 10^{-6}$ & $4.21 \times 10^{-4}$ & GNAQ; MITF; TYR; MC1R; MAPK3; MAPK1 \\
\hline Long-term depression & $8.99 \times 10^{-6}$ & $4.43 \times 10^{-4}$ & GNAQ; GNA11; BRAF; MAPK3; MAPK1 \\
\hline Glioma & $1.34 \times 10^{-5}$ & $4.94 \times 10^{-4}$ & CDK4; BRAF; MAPK3; MAPK1; CDKN2A \\
\hline Pathways in cancer & $1.18 \times 10^{-5}$ & $4.94 \times 10^{-4}$ & $\begin{array}{l}\text { GNAQ; MITF; CDK4; BRCA2; GNA11; BIRC7; } \\
\text { BRAF; MAPK3; MAPK1; CDKN2A }\end{array}$ \\
\hline Chronic myeloid leukemia & $2.36 \times 10^{-5}$ & $7.77 \times 10^{-4}$ & CDK4; BRAF; MAPK3; MAPK1; CDKN2A \\
\hline Other glycan degradation & $8.13 \times 10^{-5}$ & $2.41 \times 10^{-3}$ & NEU4; NEU2; NEU3 \\
\hline Vascular smooth muscle contraction & $2.55 \times 10^{-4}$ & $6.67 \times 10^{-3}$ & GNAQ; GNA11; BRAF; MAPK3; MAPK1 \\
\hline Long-term potentiation & $2.70 \times 10^{-4}$ & $6.67 \times 10^{-3}$ & GNAQ; BRAF; MAPK3; MAPK1 \\
\hline Thyroid cancer & $3.51 \times 10^{-4}$ & $7.99 \times 10^{-3}$ & BRAF; MAPK3; MAPK1 \\
\hline Gap junction & $8.11 \times 10^{-4}$ & $1.60 \times 10^{-2}$ & GNAQ; GNA11; MAPK3; MAPK1 \\
\hline ErbB signaling pathway & $7.77 \times 10^{-4}$ & $1.60 \times 10^{-2}$ & NCK1; BRAF; MAPK3; MAPK1 \\
\hline GnRH signaling pathway & $9.20 \times 10^{-4}$ & $1.70 \times 10^{-2}$ & GNAQ; GNA11; MAPK3; MAPK1 \\
\hline Sphingolipid metabolism & $1.47 \times 10^{-3}$ & $2.36 \times 10^{-2}$ & NEU4; NEU2; NEU3 \\
\hline Chagas disease (American trypanosomiasis) & $1.51 \times 10^{-3}$ & $2.36 \times 10^{-2}$ & GNAQ; GNA11; MAPK3; MAPK1 \\
\hline $\mathrm{T}$ cell receptor signaling pathway & $1.51 \times 10^{-3}$ & $2.36 \times 10^{-2}$ & NCK1; CDK4; MAPK3; MAPK1 \\
\hline Serotonergic synapse & $1.99 \times 10^{-3}$ & $2.68 \times 10^{-2}$ & GNAQ; BRAF; MAPK3; MAPK1 \\
\hline Endometrial cancer & $1.97 \times 10^{-3}$ & $2.68 \times 10^{-2}$ & BRAF; MAPK3; MAPK1 \\
\hline Cholinergic synapse & $1.93 \times 10^{-3}$ & $2.68 \times 10^{-2}$ & GNAQ; GNA11; MAPK3; MAPK1 \\
\hline Acute myeloid leukemia & $2.56 \times 10^{-3}$ & $3.03 \times 10^{-2}$ & BRAF; MAPK3; MAPK1 \\
\hline NOD-like receptor signaling pathway & $2.56 \times 10^{-3}$ & $3.03 \times 10^{-2}$ & CXCL1; MAPK3; MAPK1 \\
\hline Neurotrophin signaling pathway & $2.56 \times 10^{-3}$ & $3.03 \times 10^{-2}$ & MAGED1; BRAF; MAPK3; MAPK1 \\
\hline mTOR signaling pathway & $2.97 \times 10^{-3}$ & $3.25 \times 10^{-2}$ & BRAF; MAPK3; MAPK1 \\
\hline Viral carcinogenesis & $2.86 \times 10^{-3}$ & $3.25 \times 10^{-2}$ & CDK4; HLA-E; MAPK3; MAPK1; CDKN2A \\
\hline Colorectal cancer & $3.26 \times 10^{-3}$ & $3.44 \times 10^{-2}$ & BRAF; MAPK3; MAPK1 \\
\hline Natural killer cell mediated cytotoxicity & $3.92 \times 10^{-3}$ & $3.87 \times 10^{-2}$ & BRAF; HLA-E; MAPK3; MAPK1 \\
\hline Renal cell carcinoma & $3.89 \times 10^{-3}$ & $3.87 \times 10^{-2}$ & BRAF; MAPK3; MAPK1 \\
\hline
\end{tabular}

the network with significantly enriched GO terms and KEGG pathways.

Survival analysis. Associations among genes contained in the network and overall survival rates of patients with melanoma were evaluated using the GEPIA database. As a result, two genes contained in the MDSet, hedgehog acyltransferase (HHAT) and BRCA1-associated protein 1 (BAP1), and another two genes, TYR and microphthalmia-associated transcription factor (MITF), were identified to be significantly associated with the overall survival rates of patients with melanoma. Elevated expression levels of the four genes were all closely associated with poor melanoma prognosis (Fig. 4).

\section{Discussion}

The purpose of the present study was to explore the underlying mechanism of melanoma and potential gene biomarkers using comprehensive bioinformatics methods. The random walk method identified 111 potential melanoma-associated genes, and functional enrichment analysis identified several biological processes and pathways which may contribute to melanoma progression. A melanoma-specific network in combination with survival analysis identified more reliable biomarkers for melanoma diagnosis and treatment.

$\mathrm{GO}$ enrichment analysis indicated that 'developmental pigmentation', 'pigmentation' and 'pigment cell differentiation' were closely associated with melanoma-associated genes. Pigmentation is determined by the proportion of several melanin species, for instance the alkali soluble yellow-red pheomelanin and insoluble brown-black eumelanin (22). Synthesis of the two types of melanin starts with the tyrosine oxidation of TYR, which produces L-3,4-dihydroxyphenylalanine, which then produces dopaquinone and eventually the common precursor. The range of individual pigmentation phenotypes is the result of the ratio of true melanin to brown melanin. Individuals with darker hair or skin possess higher ratios of true melanin to 
A

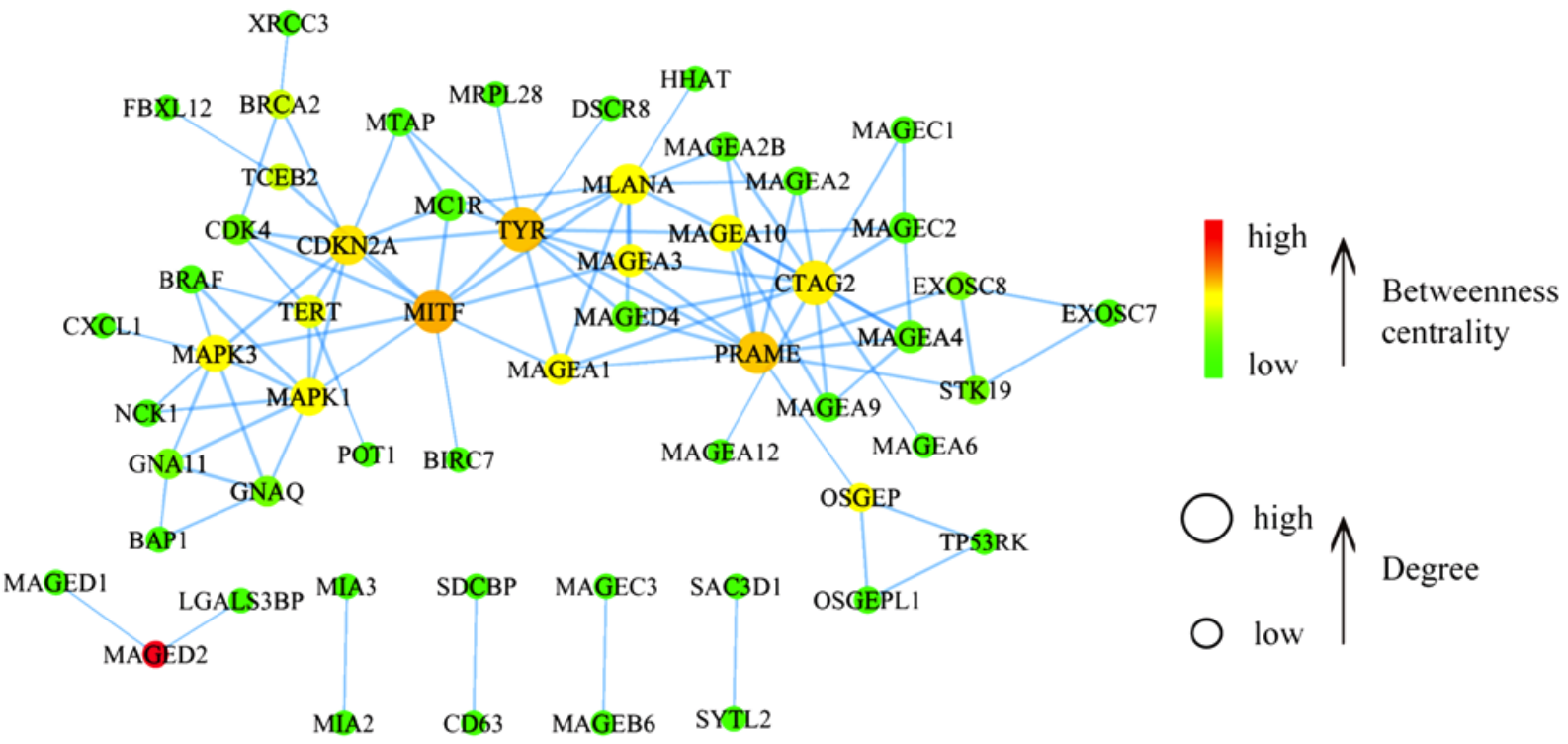

в


Figure 3. Melanoma-specific network analysis. (A) Melanoma-specific network. (B) Allocation of genes contained in the network to significantly enriched GO terms and Kyoto Encyclopedia of Genes and Genomes pathways. Function was defined according to GO terms. GO, Gene Ontology.

brown melanin than those with lighter hair or skin. Several studies have demonstrated that skin which contains brown melanin provides weak UV protection, suggesting that brown melanin may actually be a prooxidant and photosensitive to DNA damage, while eumelanin may act as a light-protective antioxidant $(23,24)$. Reactive oxygen species (ROS) may also be produced as byproducts during pigment synthesis (24). It has been considered that the role of melanocyte organelles is to provide protection against ROS-induced injury by containment (25). Leakage of toxic substances from the melanosome can be damaging to melanocytes. Additionally, pheomelanin synthesis stimulates melanoma genesis via an UV-independent pathway that induces ROS-mediated DNA damage, which may lead to melanoma carcinogenesis (26).

The deubiquitinating enzyme BAP1 is a tumor suppressor, which is inactivated in a variety of types of cancer (27). BAP1 inactivation is expected to affect transcription regulation, either through direct gene expression dysregulation or chromatin structure perturbation $(28,29)$. It has been reported that BAP1 affects ROS homeostasis and serves a major role in regulating cell morphology, cell migration and mitochondrial respiration in mesothelioma (30). In uveal melanoma, BAP1 inactivation is associated with metastasis development and poor prognosis (31). Therefore, BAP1 may promote tumor cell motility and invasiveness, and stimulates metastasis formation in vivo, which is consistent with the results of the study by Joseph et al (32).

MITF is the main regulator of melanocytes and serves a vital role in the pigmentation pathway. It also has antioxidant and prooxidant components (33). There are at least nine isoforms of MITF, which exhibit tissue-specific expression patterns, and the M isoform of MITF is selectively expressed in melanocytes (34). Melanoma is considered to arise from melanocytes, the pigment-producing cells of the skin, hair and eyes. In addition to its vital roles in normal melanocytes, MITF may also serve vital roles in melanoma, where it has been revealed to be a genetically-specific survival oncogene that is amplified in $20 \%$ of human types of melanoma (35). Previously, 



Figure 4. Kaplan-Meier curves for melanoma samples with lower (blue lines) or higher (red lines) expression levels of TYR, HHAT, BAP1 and MITF. The median expression values were used as thresholds. $\mathrm{n}=228$ for HHAT as the data for one case was not available. BAP1, BRAC1 associated protein 1; HHAT, hedgehog acyltransferase; HR, hazard ratio; MITF, melanocyte inducing transcription factor; TYR, tyrosinase.

MITF has been demonstrated to be involved in regulating metabolism and oxidative stress by directly regulating the expression of the main mitochondrial regulator; peroxisome proliferator-activated receptor $\gamma$ coactivator $1 \alpha$ (36).

The TYR gene, which encodes melanogenesis enzymes, has been associated with melanoma (37). Subsequent to identifying that variants of TYR affect pigmentation features, these same variants were revealed to be associated with the risk of skin cancer, even when accounting for available assessments of pigmentation (38). The association of TYR and its variants is robust in regulating pigmentation effects.
The hedgehog family of secreted proteins act as morphogens to control embryonic patterning and development in a variety of organ systems. HHAT mutation occurs in the conserved membrane bound $\mathrm{O}$-acyltransferase domain. HHAT has been demonstrated to be expressed in the somatic cells of XX and XY gonads at the time of sex determination, and HHAT loss of function recapitulates the majority of the testicular, skeletal, neuronal and growth defects observed in humans (39). In the developing testis, HHAT is not required for Sertoli cell commitment but serves a role in proper testis cord formation and the differentiation of fetal Leydig cells. 
Optimal BP terms were consistent with expectations, and several catabolism-associated terms were among them. Optimal features contained the terms ganglioside catabolic process, oligosaccharide catabolic process, glycosphingolipid catabolic process, ceramide catabolic process, transfer RNA threonylcarbamoyladenosine metabolic process and glycolipid catabolic process. A previous study demonstrated that numerous melanoma genes control cell metabolism, suggesting that melanoma may be a metabolic disease (40). Melanoma patterns in normal cells depend on the availability of substrates and oxygen. The regulation of metabolic pathways in cancer cells and the regulation of metabolic pathways in normal cells are significantly different. A number of studies have revealed fundamental differences in metabolic characteristics between normal melanocytes and melanoma cells in vitro (41-43). The metabolic characteristics of the two cell types were further characterized by the use of stable isotopes as tracers to quantify cell metabolic flux (44). According to the Warburg effect, the relative contribution of mitochondrial respiration to energy production in melanoma cells is generally low due to its higher glycolytic rate. In addition, melanoma cells exhibit high levels of glutaminase, which is the reverse flux of the tricarboxylic acid cycle and provides carbon for synthesis of fatty acids (44).

A total of $40-60 \%$ of cutaneous types of melanoma harbor B-Raf proto-oncogene $(B R A F)^{V 600}$ mutations (45). $B R A F$ mutations are known to constitutively activate the MAPK signaling pathway $(46,47)$. MAPK3 belongs to the MAPK family, which includes extracellular signal regulation kinases (48). When activated by upstream kinases, MAPK phosphorylates several transcription factors and serves a vital role in regulating cancer cell proliferation, differentiation and other cellular activities directly associated with cancer. A previous study revealed that the expression levels of members of the MAPK signaling pathway were altered in patients with metastatic melanoma, which may activate this signaling pathway (49).

The telomere-associated terms, including 'telomere maintenance', 'telomere organization' and 'establishment of protein localization to telomere', may also be associated with melanoma. Telomere length was considered to be a major risk factor for melanoma, increasing melanoma risk in a study of 557 cases $(50)$, and increasing nevus number $(51,52)$. In addition, high penetrative melanoma mutations were reported in genes encoding the protection of telomeres 1 (POT1) components, which are essential for telomere maintenance and signal transduction functions (53). POT1 mutations result in longer telomeres (54). A previous study investigated the mechanism of melanoma, and hypothesized that longer telomeres increase the duration of cell proliferation in a melanocytic nevus (52). If the process of aging is delayed in melanocytes, it could lead to further mutations, which increases the probability of developing a malignant tumor (50).

The thymus development process is also associated with melanoma. Interleukin-32 (IL-32) is a novel cytokine, involved in cancer development, and expressed in numerous human tissues and organs, including the thymus. Nicholl et al (55) reported that exogenous addition of IL-32 can effectively inhibit the proliferation of human melanoma cell lines, and that it is associated with increased expression of p21, p53 and tumor necrosis factor receptor superfamily member 10a. In previous studies, IL-32 has been reported to inhibit the growth and metastasis of cancer cells by regulating nuclear factor $\mathrm{\kappa B}$ (NF- $\kappa \mathrm{B})$ signaling $(56,57)$. NF- $\kappa \mathrm{B}$ is a transcription factor that regulates the expression of cytokines, growth factors and apoptosis inhibiting factors. Additionally, IL-32 is associated with signal transducer and activator of transcription 3 (STAT3) signaling in cancer cell growth (58). Numerous studies have reported that STAT3 signaling promotes cancer development, while loss of STAT3 inhibits cancer development (59-61).

In KEGG pathway analysis, certain types of cancer, including pancreatic and bladder cancer, were significantly enriched, which may indicate that melanoma has a similar phenotype, mechanism or co-morbidity with these types of cancer. Pathways, including chronic myeloid leukemia and acute myeloid leukemia, are directly associated with cancer and potential oncogenes. As a result, the abnormal expression of oncogenes may lead to cell cycle disturbances, continued cell proliferation, reduced apoptosis and tumor metastasis (62). The mTOR signaling pathway is involved in regulating cell growth, division, survival and transcription (63). Overactivation of mTOR can promote proliferation and reduce apoptosis. In addition, mTOR pathway dysfunction has been observed in several types of tumor $(64,65)$. A previous study suggested that CTAG2 is closely associated with cancer/testis antigen 1B (CTAG1B) (66). CTAG2 and CTAG1B are overexpressed in numerous types of tumor, including melanoma, sarcoma and multiple myeloma, as well as certain types of carcinoma, including lung, head and neck, and ovarian carcinoma $(66,67)$.

In the present study, TYR, HHAT, BAP1 and MITF were identified as potential therapeutic targets for melanoma. As the occurrence and development of melanoma is a complex event, the specific roles of these genes in the regulatory network remain to be determined by further experiments. Furthermore, in addition to the present study, multiple biomarkers have been identified to be associated with melanoma progression, proliferation, immune response, oncogenesis and other aspects by microarray studies $(68,69)$. As the public databases used, including OMIM and STRING, did not provide patient characteristics information, the association between the expression levels of candidate genes and clinicopathological parameters could not be assessed. Further analysis will be conducted in a future study with information from The Cancer Genome Atlas and Gene Expression Omnibus data sets. Additionally, it remains a great challenge to apply this information in a therapeutic manner.

In conclusion, in the present study, prioritization and comprehensive analysis of melanoma candidate genes were conducted to obtain several potential biomarkers and biological processes which may contribute to melanoma progression. This should be helpful in understanding the underlying mechanisms of melanoma, as well as for its diagnosis and treatment. However, further experimental validation of the results of the present study is required.

\section{Acknowledgements}

Not applicable.

\section{Funding}

No funding was received. 


\section{Availability of data and materials}

All data generated and/or analyzed during this study are included in this published article.

\section{Authors' contributions}

$\mathrm{CF}$ and $\mathrm{MB}$ made substantial contributions to the conception and design of the study. $\mathrm{CF}, \mathrm{HZ}$ and $\mathrm{AZ}$ participated in the acquisition, analysis and interpretation of data. $\mathrm{MB}, \mathrm{HZ}$ and WZ have been involved in drafting the manuscript or revising it critically for important intellectual content. WZ participated in the data analysis. CF and MB gave final approval of the version to be published. $\mathrm{CF}$ and $\mathrm{MB}$ have agreed to be accountable for all aspects of the work in ensuring that questions related to the accuracy or integrity of any part of the work are appropriately investigated and resolved.

\section{Ethics approval and consent to participate}

Not applicable.

\section{Patient consent for publication}

Not applicable.

\section{Competing interests}

The authors declare that they have no competing interests.

\section{References}

1. MacKie RM, Hauschild A and Eggermont AM: Epidemiology of invasive cutaneous melanoma. Ann Oncol 20 (Suppl 6): vi1-7, 2009.

2. Siegel RL, Miller KD and Jemal A: Cancer statistics, 2019. CA Cancer J Clin 69: 7-34, 2019.

3. Caini S, Gandini S, Sera F, Raimondi S, Fargnoli MC, Boniol M and Armstrong BK: Meta-analysis of risk factors for cutaneous melanoma according to anatomical site and clinico-pathological variant. Eur J Cancer 45: 3054-3063, 2009.

4. Ingraffea A: Melanoma. Facial Plast Surg Clin North Am 21: 33-42, 2013.

5. Owens B: Melanoma. Nature 515: S109, 2014.

6. Markovic SN, Erickson LA, Rao RD, Weenig RH, Pockaj BA, Bardia A, Vachon CM, Schild SE, McWilliams RR, Hand JL, et al: Malignant melanoma in the 21st century, part 1 : Epidemiology, risk factors, screening, prevention, and diagnosis Mayo Clin Proc 82: 364-380, 2007.

7. Rigel DS: Epidemiology of melanoma. Semin Cutan Med Surg 29: 204-209, 2010.

8. Gandini S, Sera F, Cattaruzza MS, Pasquini P, Picconi O, Boyle P and Melchi CF: Meta-analysis of risk factors for cutaneous melanoma: II. Sun exposure. Eur J Cancer 41: 45-60, 2005.

9. Elwood JM and Jopson J: Melanoma and sun exposure: An overview of published studies. Int J Cancer 73: 198-203, 1997.

10. Stern RS; PUVA Follow up Study: The risk of melanoma in association with long-term exposure to PUVA. J Am Acad Dermatol 44: 755-761, 2001.

11. Bevona C, Goggins W, Quinn T, Fullerton $J$ and Tsao H: Cutaneous melanomas associated with nevi. Arch Dermatol 139: 1620-1624, 2003.

12. Tsao H and Niendorf K: Genetic testing in hereditary melanoma. J Am Acad Dermatol 51: 803-808, 2004

13. Stam-Posthuma JJ, van Duinen C, Scheffer E, Vink J and Bergman W: Multiple primary melanomas. J Am Acad Dermatol 44: 22-27, 2001.

14. Hanauer DA, Rhodes DR, Sinha-Kumar C and Chinnaiyan AM Bioinformatics approaches in the study of cancer. Curr Mol Med 7: 133-141, 2007
15. Cho A, Shim JE, Kim E, Supek F,Lehner B and Lee I: MUFFINN: Cancer gene discovery via network analysis of somatic mutation data. Genome Biol 17: 129, 2016.

16. Ding KF, Finlay D, Yin H, Hendricks WPD, Sereduk C, Kiefer J, Sekulic A, LoRusso PM, Vuori K, Trent JM and Schork NJ: Network rewiring in cancer: Applications to melanoma cell lines and the cancer genome atlas patients. Front Genet 9: 228, 2018.

17. Amberger JS, Bocchini CA, Schiettecatte F, Scott AF and Hamosh A: OMIM.org: Online mendelian inheritance in man $(\mathrm{OMIM}(\mathrm{R}))$, an online catalog of human genes and genetic disorders. Nucleic Acids Res 43: D789-D798, 2015.

18. Dembélé D: Analysis of high-throughput biological data using their rank values. Stat Methods Med Res 96228021876418, 2018 (Epub ahead of print).

19. Wang J, Duncan D, Shi Z and Zhang B: WEB-based GEne SeT AnaLysis Toolkit (WebGestalt): Update 2013. Nucleic Acids Res 41: W77-W83, 2013.

20. Abbas A, Kong XB, Liu Z, Jing BY and Gao X: Automatic peak selection by a Benjamini-Hochberg-based algorithm. PLoS One 8: e53112, 2013.

21. Khuri S and Wuchty S: Essentiality and centrality in protein interaction networks revisited. BMC Bioinformatics 16: 109, 2015.

22. Ito $\mathrm{S}$ and Wakamatsu K: Chemistry of mixed melanogenesis-pivotal roles of dopaquinone. Photochem Photobiol 84: 582-592, 2008.

23. Samokhvalov A, Hong L, Liu Y, Garguilo J, Nemanich RJ, Edwards GS and Simon JD: Oxidation potentials of human eumelanosomes and pheomelanosomes. Photochem Photobiol 81: $145-148,2005$.

24. Meredith P and Sarna T: The physical and chemical properties of eumelanin. Pigment Cell Res 19: 572-594, 2006.

25. Chen KG, Valencia JC, Gillet JP, Hearing VJ and Gottesman MM: Involvement of ABC transporters in melanogenesis and the development of multidrug resistance of melanoma. Pigment Cell Melanoma Res 22: 740-749, 2009.

26. Montano SP, Pigli YZ and Rice PA: The mu transpososome structure sheds light on DDE recombinase evolution. Nature 491: 413-417, 2012.

27. Wiesner T, Murali R, Fried I, Cerroni L, Busam K, Kutzner H and Bastian BC: A distinct subset of atypical Spitz tumors is characterized by BRAF mutation and loss of BAP1 expression. Am J Surg Pathol 36: 818-830, 2012.

28. Dey A, Seshasayee D, Noubade R, French DM, Liu J, Chaurushiya MS, Kirkpatrick DS, Pham VC, Lill JR, Bakalarski CE, et al: Loss of the tumor suppressor BAP1 causes myeloid transformation. Science 337: 1541-1546, 2012.

29. Baymaz HI, Fournier A, Laget S, Ji Z, Jansen PW, Smits AH, Ferry L, Mensinga A, Poser I, Sharrocks A, et al: MBD5 and MBD6 interact with the human PR-DUB complex through their methyl-CpG-binding domain. Proteomics 14: 2179-2189, 2014.

30. Hebert L, Bellanger D, Guillas C, Campagne A, Dingli F, Loew D, Fievet A, Jacquemin V, Popova T, Jean D, et al: Modulating BAP1 expression affects ROS homeostasis, cell motility and mitochondrial function. Oncotarget 8: 72513-72527, 2017.

31. Harbour JW, Onken MD, Roberson ED, Duan S, Cao L, Worley LA, Council ML, Matatall KA, Helms C, Bowcock AM: Frequent mutation of BAP1 in metastasizing uveal melanomas. Science 330: 1410-1413, 2010.

32. Joseph RW, Kapur P, Serie DJ, Eckel-Passow JE, Parasramka M, Ho T, Cheville JC, Frenkel E, Rakheja D, Brugarolas J and Parker A: Loss of BAP1 protein expression is an independent marker of poor prognosis in patients with low-risk clear cell renal cell carcinoma. Cancer 120: 1059-1067, 2014.

33. Hershey CL and Fisher DE: Genomic analysis of the microphthalmia locus and identification of the MITF-J/Mitf-J isoform. Gene 347: 73-82, 2005.

34. Fuse N, Yasumoto K, Suzuki H, Takahashi K and Shibahara S: Identification of a melanocyte-type promoter of the microphthalmia-associated transcription factor gene. Biochem Biophys Res Commun 219: 702-707, 1996.

35. Garraway LA, Widlund HR, Rubin MA, Getz G, Berger AJ, Ramaswamy S, Beroukhim R, Milner DA, Granter SR, Du J, et al: Integrative genomic analyses identify MITF as a lineage survival oncogene amplified in malignant melanoma. Nature 436: 117-122, 2005.

36. Haq R, Shoag J, Andreu-Perez P, Yokoyama S, Edelman H, Rowe GC, Frederick DT, Hurley AD, Nellore A, Kung AL, et al: Oncogenic BRAF regulates oxidative metabolism via PGC1a and MITF. Cancer Cell 23: 302-315, 2013. 
37. Gudbjartsson DF, Sulem P, Stacey SN, Goldstein AM, Rafnar T, Sigurgeirsson B, Benediktsdottir KR, Thorisdottir K, Ragnarsson R, Sveinsdottir SG, et al: ASIP and TYR pigmentation variants associate with cutaneous melanoma and basal cell carcinoma. Nat Genet 40: 886-891, 2008.

38. Saternus R, Pilz S, Gräber S, Kleber M, März W, Vogt T and Reichrath J: A closer look at evolution: Variants (SNPs) of genes involved in skin pigmentation, including EXOC2, TYR, TYRP1, and DCT, are associated with $25(\mathrm{OH}) \mathrm{D}$ serum concentration. Endocrinology 156: 39-47, 2015.

39. Callier P, Calvel P, Matevossian A, Makrythanasis P, Bernard P, Kurosaka H, Vannier A, Thauvin-Robinet C, Borel C, Mazaud-Guittot S, et al: A Loss of Function mutation in the palmitoyl-transferase HHAT Leads to Syndromic 46,XY disorder of sex development by impeding hedgehog protein palmitoylation and signaling. PLoS Genet 10: e1004340, 2014.

40. Abildgaard $C$ and Guldberg P: Molecular drivers of cellular metabolic reprogramming in melanoma. Trends Mol Med 21: 164-171, 2015.

41. Abildgaard C, Dahl C, Basse AL, Ma T and Guldberg P: Bioenergetic modulation with dichloroacetate reduces the growth of melanoma cells and potentiates their response to BRAFV600E inhibition. J Transl Med 12: 247, 2014.

42. Barbi de Moura M, Vincent G, Fayewicz SL, Bateman NW, Hood BL, Sun M, Suhan J, Duensing S, Yin Y, Sander C, et al: Mitochondrial respiration-an important therapeutic target in melanoma. PLoS One 7: e40690, 2012.

43. Hall A, Meyle KD, Lange MK, Klima M, Sanderhoff M, Dahl C, Abildgaard C, Thorup K, Moghimi SM, Jensen PB, et al: Dysfunctional oxidative phosphorylation makes malignant melanoma cells addicted to glycolysis driven by the (V600E)BRAF oncogene. Oncotarget 4: 584-599, 2013.

44. Scott DA, Richardson AD, Filipp FV, Knutzen CA, Chiang GG Ronai ZA, Osterman AL and Smith JW: Comparative metabolic flux profiling of melanoma cell lines: Beyond the Warburg effect. J Biol Chem 286: 42626-42634, 2011.

45. Dong J, Phelps RG, Qiao R, Yao S, Benard O, Ronai Z and Aaronson SA: BRAF oncogenic mutations correlate with progression rather than initiation of human melanoma. Cancer Res 63: 3883-5, 2003

46. Pflugfelder A, Kochs C, Blum A, Capellaro M, Czeschik C, Dettenborn T, Dill D, Dippel E, Eigentler T, Feyer P, et al: Malignant melanoma S3-guideline 'diagnosis, therapy and follow-up of melanoma'. J Dtsch Dermatol Ges 11 (Suppl 6) S1-S126, 2013

47. Stadler S, Weina K, Gebhardt C and Utikal J: New therapeutic options for advanced non-resectable malignant melanoma. Adv Med Sc 60: 83-88, 2015.

48. Garcia F, Zalba G, Paez G, Encio I and de Miguel C: Molecular cloning and characterization of the human p44 mitogen-activated protein kinase gene. Genomics 50: 69-78, 1998.

49. Orouji E, Orouji A, Gaiser T, Larribere L, Gebhardt C and Utikal J: MAP kinase pathway gene copy alterations in NRAS/BRAF wild-type advanced melanoma. Int J Cancer 138: 2257-2262, 2016.

50. Nan H, Du M, De Vivo I, Manson JE, Liu S, McTiernan A, Curb JD, Lessin LS, Bonner MR, Guo Q, et al: Shorter telomeres associate with a reduced risk of melanoma development. Cancer Res 71: 6758-6763,2011.

51. Han J, Qureshi AA, Prescott J, Guo Q, Ye L, Hunter DJ and De Vivo I: A prospective study of telomere length and the risk of skin cancer. J Invest Dermatol 129: 415-421, 2009.

52. Bataille V, Kato BS, Falchi M, Gardner J, Kimura M, Lens M, Perks U, Valdes AM, Bennett DC, Aviv A and Spector TD Nevus size and number are associated with telomere length and represent potential markers of a decreased senescence in vivo. Cancer Epidemiol Biomarkers Prev 16: 1499-1502, 2007.

53. Horn S, Figl A, Rachakonda PS, Fischer C, Sucker A, Gast A, Kadel S, Moll I, Nagore E, Hemminki K, et al: TERT promoter mutations in familial and sporadic melanoma. Science 339: 959-961, 2013.
54. Robles-Espinoza CD, Harland M, Ramsay AJ, Aoude LG, Quesada V, Ding Z1, Pooley KA, Pritchard AL, Tiffen JC, Petljak M, et al: POT1 loss-of-function variants predispose to familial melanoma. Nat Genet 46: 478-481, 2014.

55. Nicholl MB, Chen X, Qin C, Bai Q, Zhu Z, Davis MR and Fang Y: IL-32 $\alpha$ has differential effects on proliferation and apoptosis of human melanoma cell lines. J Surg Oncol 113: 364-369, 2016

56. Tsai CY, Wang CS, Tsai MM, Chi HC, Cheng WL, Tseng YH, Chen CY, Lin CD, Wu JI, Wang LH and Lin KH: Interleukin-32 increases human gastric cancer cell invasion associated with tumor progression and metastasis. Clin Cancer Res 20: 2276-2288, 2014

57. Yun HM, Oh JH, Shim JH, Ban JO, Park KR, Kim JH, Lee DH, Kang JW, Park YH, Yu D, et al: Antitumor activity of IL-32 $\beta$ through the activation of lymphocytes, and the inactivation of NF- $\kappa \mathrm{B}$ and STAT3 signals. Cell Death Dis 4: e640, 2013

58. Oh JH, Cho MC, Kim JH, Lee SY, Kim HJ, Park ES, Ban JO, Kang JW, Lee DH, Shim JH, et al: IL-32 $\gamma$ inhibits cancer cell growth through inactivation of NF- $\mathrm{kB}$ and STAT3 signals. Oncogene 30: 3345-3359, 2011.

59. Chan KS, Sano S, Kiguchi K, Anders J, Komazawa N, Takeda J and DiGiovanni J: Disruption of Stat3 reveals a critical role in both the initiation and the promotion stages of epithelial carcinogenesis. J Clin Invest 114: 720-728, 2004.

60. Jenkins BJ, Roberts AW, Najdovska M, Grail D and Ernst M: The threshold of gp130-dependent STAT3 signaling is critical for normal regulation of hematopoiesis. Blood 105: 3512-3520, 2005.

61. Lee H, Herrmann A, Deng JH, Kujawski M, Niu G, Li Z, Forman S, Jove R, Pardoll DM and Yu H: Persistently activated Stat3 maintains constitutive NF-kappaB activity in tumors. Cancer Cell 15: 283-293, 2009.

62. Arvanitis C and Felsher DW: Conditional transgenic models define how MYC initiates and maintains tumorigenesis. Semin Cancer Biol 16: 313-317, 2006.

63. Hay N and Sonenberg N: Upstream and downstream of mTOR. Genes Dev 18: 1926-1945, 2004.

64. Beevers CS, Li F, Liu L and Huang S: Curcumin inhibits the mammalian target of rapamycin-mediated signaling pathways in cancer cells. Int J Cancer 119: 757-764, 2006.

65. Matsubara S, Ding Q, Miyazaki Y, Kuwahata T, Tsukasa K and Takao S: mTOR plays critical roles in pancreatic cancer stem cells through specific and stemness-related functions. Sci Rep 3: 3230, 2013.

66. Lethe B, Lucas S, Michaux L, De Smet C, Godelaine D, Serrano A, De Plaen E and Boon T: LAGE-1, a new gene with tumor specificity. Int J Cancer 76: 903-908, 1998.

67. Nicholaou T, Ebert L, Davis ID, Robson N, Klein O, Maraskovsky E, Chen W and Cebon J: Directions in the immune targeting of cancer: Lessons learned from the cancer-testis Ag NY-ESO-1. Immunol Cell Biol 84: 303-317, 2006.

68. Vizkeleti L, Kiss T, Koroknai V, Ecsedi S, Papp O, Szasz I, Adany R and Balazs M: Altered integrin expression patterns shown by microarray in human cutaneous melanoma. Melanoma Res 27: 180-188, 2017.

69. Wozniak M, Peczek L, Czernek L and Düchler M: Analysis of the miRNA profiles of melanoma exosomes derived under normoxic and hypoxic culture conditions. Anticancer Res 37: 6779-6789, 2017

This work is licensed under a Creative Commons Attribution-NonCommercial-NoDerivatives 4.0 International (CC BY-NC-ND 4.0) License. 